\title{
LOCAL MEASUREMENT OF $\Lambda$ USING PULSAR TIMING ARRAYS
}

\author{
DOMÈnec EsPriu AND DANiEl PUIGDOMÈnech \\ Departament d'Estructura i Constituents de la Matèria and Institut de Ciències del Cosmos (ICCUB), \\ Universitat de Barcelona, Martí i Franquès 1, E-08028 Barcelona, Spain \\ Received 2012 July 31; accepted 2012 December 10; published 2013 February 4
}

\begin{abstract}
We consider the propagation of gravitational waves (GWs) in de Sitter spacetime and how a non-zero value of the cosmological constant might affect their detection in pulsar timing arrays (PTAs). If $\Lambda \neq 0$, the waves are anharmonic in Friedmann-Robertson-Walker coordinates, and although this effect is very small it gives rise to noticeable consequences for GWs originating in extragalactic sources such as spiraling black hole binaries. The results indicate that the timing residuals induced by GWs from such sources in PTAs will show a peculiar angular dependence with a marked enhancement around a particular value of the angle subtended by the source and the pulsars, depending mainly on the actual value of the cosmological constant and the distance to the source. The position of the peak could represent a gauge of the value of $\Lambda$. The enhancement that the new effect brings about could facilitate the first direct detection of GWs while representing a local measurement of $\Lambda$.
\end{abstract}

Key words: cosmological parameters - dark energy - gravitational waves - pulsars: general

Online-only material: color figures

\section{INTRODUCTION}

Pulsar timing arrays (PTAs) are among the most promising candidates to provide the first direct detection of gravitational waves (GWs). They have already been collecting data for almost a decade and they are expected to obtain signals in the next few years. The idea behind the PTA is to detect the correlated disruption of the periods measured for a significant number of pulsars due to the passing of a GW through the system (Hobbs 2008a, 2008b, 2011; Hobbs et al. 2010; Lee et al. 2011). The frequency range sensitive to this method is $10^{-9} \mathrm{~s}^{-1} \leqslant w \leqslant 10^{-7} \mathrm{~s}^{-1}$ (Hobbs 2011), and the timing residual is expected to follow a power law (Hobbs 2008a, 2008b; Jenet et al. 2006). A key problem in making predictions for these signals is modeling in a realistic way the wavefunctions produced in the different sources; in particular, the value of the amplitude of the metric perturbation $h$ is a free parameter in principle. Some bounds in the range of $10^{-17} \leqslant h \leqslant 10^{-15}$ have been already set (Jenet et al. 2006).

If $\Lambda \neq 0$, GWs propagate in a de Sitter spacetime, not in flat Minkowskian spacetime. The general practice is simply to account for the expansion of the universe by using a redshifted frequency according to the distance of the source (Lee et al. 2011). In this work we go beyond this exceedingly simple approximation and use an approximate solution of the GW equation in de Sitter spacetime, previously derived by Bernabeu et al. (2011), and see that the conclusions change.

We assume that $\Lambda$ is somehow an intrinsic property of spacetime rather than an effective description valid at extremely large scales. If so, it is expected to be present at virtually all scales, with the possible exception of gravitationally bound objects such as galaxies or local groups of galaxies. If $\Lambda$ is a fundamental constant of nature, there should be a way of determining its value locally. By "locally" here we mean at redshifts $z \ll 1$. This question has been addressed by Sereno \& Jetzer (2006), Balaguera-Antolinez et al. (2006), Iorio (2008a, 2008b), Suto (1993, 1996), Peebles (1976, 1980), Adkins et al. (2007), Rindler (1998, 2006), Kottler (1918), and Bernabeu et al. (2010) with varying conclusions. We will see that GWs might open an effective window to realize this program. In fact, our results suggest that the currently observed non-zero value of $\Lambda$ may actually facilitate the first direct detection of GWs under certain circumstances.

This paper is organized as follows. In Section 2 we present the wavefunctions used, define the way in which the timing residuals are calculated, and include a brief explanation of the coordinate systems involved. Section 3 is devoted to presenting our numerical analysis. In Section 4 we discuss the possibility of using this method to obtain some results on the value of the cosmological constant. We sum up our conclusions in Section 5.

\section{GRAVITATIONAL WAVES AND TIMING RESIDUALS WITH $\Lambda \neq 0$}

In Minkowski spacetime, GWs obey the simple wave equation $\square h=0$. It is possible to show (Bernabeu et al. 2011) that in de Sitter spacetime with $\Lambda \neq 0$ and within the linearized approximation one can find solutions of the linearized Einstein equations in the traceless Lorenz gauge (TT gauge; Price \& Yang 2008) which obey the same equation of motion:

$$
\square h_{\mu \nu}^{\mathrm{SdS}}=0 .
$$

Spherical massless waves are solutions of this equation away from the source:

$$
h_{\mu \nu}^{\mathrm{SdS}}=\frac{1}{r}\left(E_{\mu \nu} \cos [w(t-r)]+D_{\mu \nu} \sin [w(t-r)]\right) .
$$

However, as shown in Bernabeu et al. (2011), this simple linearized solution only holds in a specific set of coordinates closely related to the Schwarzschild-de Sitter (SdS) coordinates. This is easily seen by considering a linearized background solution (rather than wave-like solutions) and realizing that their unique static solution is the (linearized) SdS metric (Bernabeu et al. 2011).

Although they provide a perfectly valid solution for GWs, SdS coordinates are not adequate for making observational predictions. The proper isotropic and homogeneous coordinates 


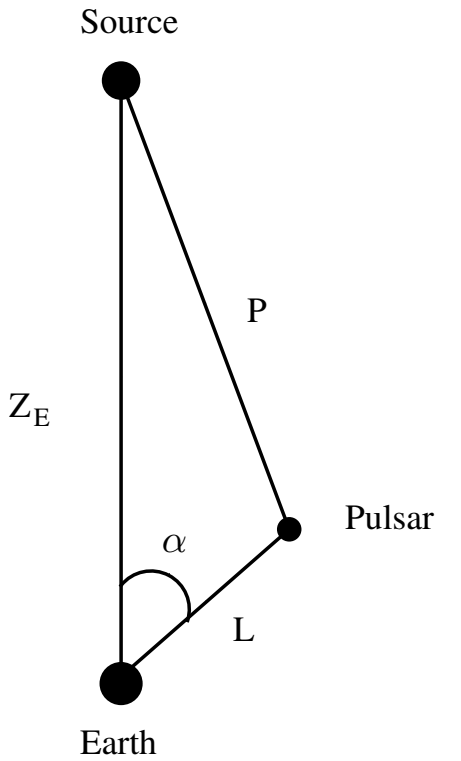

Figure 1. Relative coordinates of the GW source $(R=0)$, the Earth (located at $Z=Z_{E}$ ) with respect to the GW source, and the pulsar located at coordinates $\boldsymbol{P}=\left(P_{X}, P_{Y}, P_{Z}\right)$ with respect to the source. The $Z$ direction is chosen to be defined by the source-Earth axis. Angles $\alpha$ and $\beta$ are the polar and azimuthal angles of the pulsar with respect to this axis.

are the Friedmann-Robertson-Walker (FRW) ones $^{1}$ and the solution (2) in such coordinates, neglecting $\mathcal{O}(\Lambda)$ and higher terms, reads

$$
\begin{aligned}
h_{\mu \nu}^{\mathrm{FRW}}= & \frac{E_{\mu \nu}}{R}\left(1+\sqrt{\frac{\Lambda}{3}} T\right) \\
& \times \cos \left[w(T-R)+w \sqrt{\frac{\Lambda}{3}}\left(\frac{R^{2}}{2}-T R\right)\right] \\
& +\frac{D_{\mu \nu}}{R}\left(1+\sqrt{\frac{\Lambda}{3}} T\right) \\
& \times \sin \left[w(T-R)+w \sqrt{\frac{\Lambda}{3}}\left(\frac{R^{2}}{2}-T R\right)\right],
\end{aligned}
$$

where $R$ is the usual radial FRW comoving coordinate and $T$ is cosmological time. Note that the linearization process that has been used makes sense as long as $\Lambda T^{2}, \Lambda R^{2} \ll 1$ and also that, in the TT gauge, the only spatial components of the metric that are different from zero are the $X, Y$ entries of the polarization tensors $E_{\mu \nu}, D_{\mu \nu}$. Although some temporal components of $E_{\mu \nu}$ and $D_{\mu \nu}$ are also non-zero in these coordinates, they are several orders of magnitude smaller than the spatial ones and therefore will not be relevant for the present study.

We note that the phase velocity of propagation of the GW in such coordinates is not $v_{p}=1$ but $v_{p} \sim 1-\sqrt{(\Lambda / 3)} T+\mathcal{O}(\Lambda)$ (Bernabeu et al. 2011). On the other hand, with respect to the ruler distance traveled (computed with $g_{i j}$ ), the velocity is still 1 (up to terms in $\Lambda$ of higher order than those considered).

Consider the set-up depicted in Figure 1 describing the relative situation of a GW source (possibly a very massive black hole binary), the Earth, and a nearby pulsar. The timing residual (Deng \& Finn 2011) induced by

\footnotetext{
1 Note that the FRW metric cannot be approximated to obey any linearized
} Einstein equation; see Bernabeu et al. (2011) for a detailed discussion.
Equation (3) will be given by

$$
\begin{aligned}
& H\left(T_{E}, L, \alpha, \beta, Z_{E}, w, \varepsilon, \Lambda\right)= \\
& \quad-\frac{L}{2 c} \hat{n}^{i} \hat{n}^{j} \int_{-1}^{0} d x h_{i j}^{\mathrm{FRW}}\left(T_{E}+\frac{L}{c} x, \boldsymbol{P}+L(1+x) \hat{n}\right)
\end{aligned}
$$

along the null geodesic from the pulsar to the Earth, where we assume $^{2} \varepsilon \sim\left|E_{i j}\right| \sim\left|D_{i j}\right|, i, j=X, Y$ and the unit vector $\hat{n}$ is given by $(-\sin \alpha \cos \beta,-\sin \alpha \sin \beta, \cos \alpha), Z_{E}$ is the distance from the Earth to the source, $L$ is the distance to the pulsar, and $T_{E}$ is the time of arrival of the wave to the local system. In deriving the previous timing residual we have neglected the (non-zero) time components of $E_{\mu \nu}, D_{\mu \nu}$ that, as previously indicated, are several orders of magnitude smaller. The speed of light has been restored. We have assumed that from the pulsar to the Earth the electromagnetic signal follows the trajectory given by the line of sight $\boldsymbol{R}(x)=\boldsymbol{P}+L(1+x) \hat{n}$. Since we assume ${ }^{2}$ that within the Galaxy $\Lambda=0, L$ is also the ruler distance. Explicitly

$$
\begin{aligned}
\boldsymbol{R}(x) & =\boldsymbol{P}+L(1+x) \hat{n} \\
& =\left(-x L \sin \alpha \cos \beta,-x L \sin \alpha \sin \beta, Z_{E}+x L \cos \alpha\right)
\end{aligned}
$$

or in modulus

$$
R(x)=\sqrt{Z_{E}^{2}+2 x L Z_{E} \cos \alpha+x^{2} L^{2}} \simeq Z_{E}+x L \cos \alpha,
$$

since we are considering $L \ll Z_{E}$. This approximation does not affect in any significant way the results below, nor do we consider the known contribution to the timing residual $H$ from Earth's peculiar motion. The integral is of course independent of the angle $\beta$ for any single pulsar but it will depend on the relative angles when several pulsars are averaged.

Let us consider the arguments of the trigonometric functions in Equation (3) and define

$$
\begin{aligned}
& \Theta\left(x, T_{E}, L, \alpha, \beta, Z_{E}, w, \Lambda\right) \\
& \equiv w\left(T_{E}+\frac{L}{c} x-\frac{Z_{E}}{c}-x \frac{L}{c} \cos \alpha\right) \\
& \quad+w \sqrt{\frac{\Lambda}{3}}\left(\frac{\left(\frac{Z_{E}}{c}+x \frac{L}{c} \cos \alpha\right)^{2}}{2}-\left(T_{E}+\frac{L}{c} x\right)\right. \\
& \left.\quad \times\left(\frac{Z_{E}}{c}+x \frac{L}{c} \cos \alpha\right)\right) .
\end{aligned}
$$

Then

$$
\begin{aligned}
& H\left(T_{E}, L, \alpha, \beta, Z_{E}, w, \varepsilon, \Lambda\right) \\
& =-\frac{1}{2} \frac{L \varepsilon}{c}\left(\sin ^{2} \alpha \cos ^{2} \beta+2 \sin \alpha \sin \beta \cos ^{2} \beta-\sin ^{2} \alpha \sin ^{2} \beta\right) \\
& \quad \times \int_{-1}^{0} d x \frac{1}{\left(Z_{E}+x L \cos \alpha\right)}\left(1+\sqrt{\frac{\Lambda}{3}}\left(T_{E}+\frac{L}{c} x\right)\right) \\
& \quad \times(\cos \Theta+\sin \Theta) .
\end{aligned}
$$

At this point one should ask whether the observationally preferred, exceedingly small value of the cosmological constant (Riess et al. 1998, 2004; Perlmutter et al. 1999; Riess 2000; Linder \& Perlmutter 2007; Kowalski et al. 2008; Wood-Vasey

\footnotetext{
2 This approximation is inessential and can be easily discarded.
} 

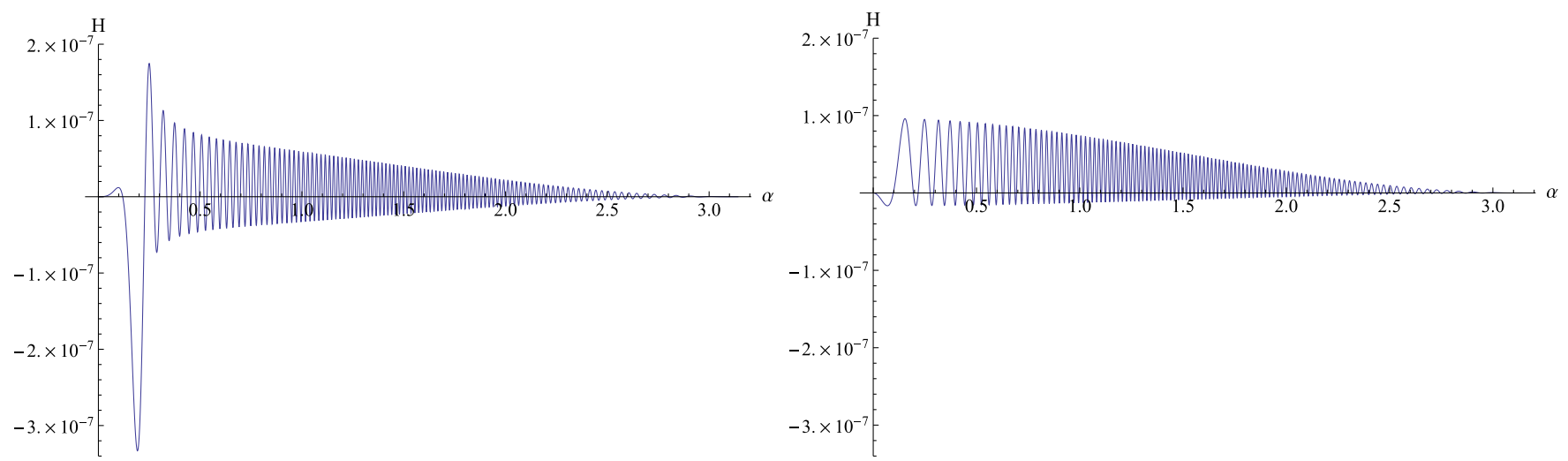

Figure 2. (Left) The raw timing residual for $\Lambda=10^{-35} \mathrm{~s}^{-2}$ as a function of the angle $\alpha$ subtended by the source and the measured pulsar as seen from the observer. The figures are symmetrical for $\pi \leqslant \alpha \leqslant 2 \pi$. (Right) The same timing residual for $\Lambda=0$. In both cases we take $\varepsilon=1.2 \times 10^{9} \mathrm{~m}, L=10^{19} \mathrm{~m}$, and $T_{E}=\left(Z_{E} / c\right) \mathrm{s}$ for $Z_{E}=3 \times 10^{24} \mathrm{~m}$; with these values $|h| \sim(\varepsilon / R) \sim 10^{-15}$ which is within the expected accuracy of PTA (Jenet et al. 2006). Similar results are obtained for other close values of $T_{E}$.

(A color version of this figure is available in the online journal.)

et al. 2007; Riess \& Livio 2006; Spergel et al. 2007; Eisenstein et al. 2005; Seo \& Eisenstein 2003; Fu et al. 2008; Guzzo et al. 2008) affects the timing residuals from a pulsar at all. To answer this question we take reasonable values of the parameters both for the GW and one pulsar location and plot a snapshot of the resulting timing residuals as a function of the angle $\alpha$ for the time of arrival, $T_{E}$, of the wave to the local system. The comparison is shown in Figure 2. The figure speaks for itself and strongly suggests that the angular dependence of the timing residual is influenced by the value of the cosmological constant, in spite of its small value. A feature that catches the eye immediately is an enhancement of the signal for a specific small angle $\alpha$ (corresponding generally to a source of low Galactic latitude, or a pulsar nearly aligned (but not quite as otherwise $E_{i j} \hat{n}^{i} \hat{n}^{j}=0$, although the total timing residual is non-vanishing due to the $\mathcal{O}(\Lambda)$ time components for TT waves) with the source.

To understand this enhancement let us analyze the behavior of the integral

$$
I=\int_{-1}^{0} d x(\cos \Theta+\sin \Theta)
$$

with $\Theta$ defined in Equation (7), as the prefactors in Equation (8) are not relevant for the discussion. The result can be expressed as a combination of Fresnel functions, and sines and cosines. In the limit where $\Lambda \rightarrow 0$ the Fresnel functions approach a constant and the behavior is the usual for trigonometric functions. In this respect, the Fresnel functions are responsible for the position and magnitude of the enhancement. This is clearly seen when $I^{2}$ is plotted ${ }^{3}$ as a function of the angular separation $\alpha$ between the source and the pulsar. $I^{2}$ always shows a maximum, the position of which is quite stable under changes of most of the parameters involved. It turns out to only depend strongly on the value of $\Lambda$ and on the distance to the source. It actually depends on the timescales involved rather than on the distance to the source but since the time of arrival of the wave to the local system is directly related to the distance, the dependence is correlated. This is evidenced in Figure 3, which shows plots of $I^{2}$ for different values of frequency, distance to the pulsar, distance to the source and cosmological constant. In Figure 3(a) the following reasonable values $Z_{E}=3 \times 10^{24} \mathrm{~m}, w=10^{-8} \mathrm{~s}^{-1}$,

3 We plot $I^{2}$ rather than $I$ to deal with a positive quantity.
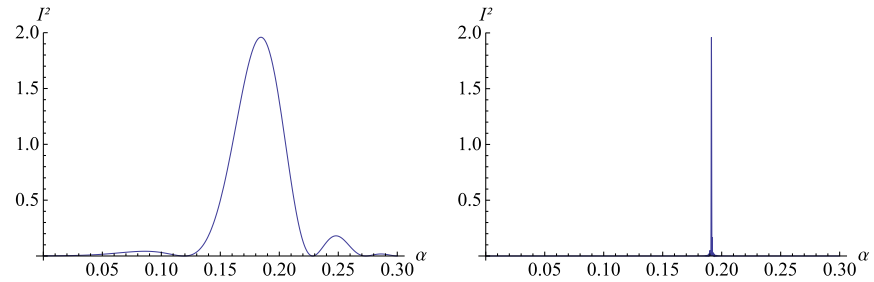

(a)

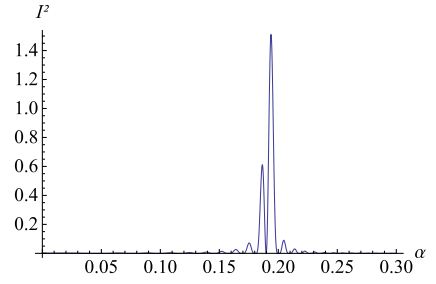

(c)

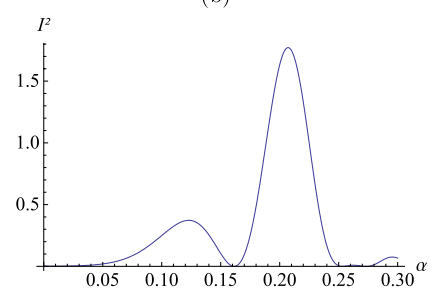

(d)

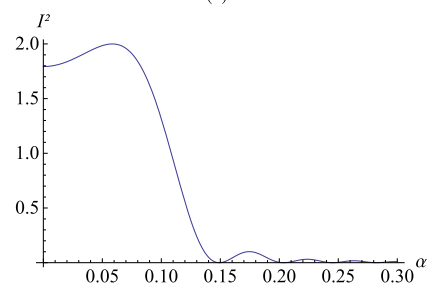

(e)

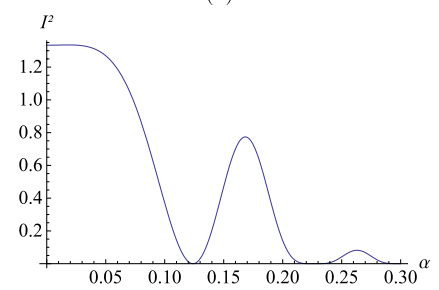

(f)

Figure 3. Integral $I^{2}$ plotted for different values of the parameters involved. (a) corresponds to the reasonable values $w=10^{-8} \mathrm{~s}^{-1}, L=10^{19} \mathrm{~m}$, $Z_{E}=3 \times 10^{24} \mathrm{~m}, \Lambda=10^{-35} \mathrm{~s}^{-2}$, and $T_{E}=10^{16} \mathrm{~s}$; (b) change in pulsar distance to $L=10^{21} \mathrm{~m}$; (c) change in frequency to $w=10^{-7} \mathrm{~s}^{-1}$; (d) change in time to $T_{E}=\left(10^{16}+10^{8}\right) \mathrm{s}$; (e) change in time and distance to the source to $Z_{E}=3 \times 10^{23} \mathrm{~m}$ and $T_{E}=10^{15} \mathrm{~s}$; (f) change in the cosmological constant to $\Lambda=10^{-36} \mathrm{~s}^{-2}$.

(A color version of this figure is available in the online journal.)

$T_{E}=\left(Z_{E} / c\right) \mathrm{s}$, and $L=10^{19} \mathrm{~m}$ are used. In (b) there is a change in the distance to the pulsar; in (c) we change the frequency; in (d) we keep the distance to the source fixed and use the time at the end of a hypothetical three-year observation; in (e) we change the distance to the source by one order of magnitude (therefore time also changes); finally in (f) the cosmological constant is changed. It is clear that the most dramatic changes occur when either the distance to the source or the value of the cosmological constant is modified. 


\section{SIGNIFICANCE OF THE TIMING RESIDUALS}

Now we would like to make a more detailed study of this possible signal. For that we use the ATNF pulsar catalogue (Manchester et al. 2005). As is well known, pulsars are remarkably stable clocks whose periods are known to a very high accuracy, up to $10^{-14} \mathrm{~s}$ in some cases. However, to achieve this extreme precision requires some hypotheses that are not appropriate for the physical situation we are considering and we will assume the more modest precision of $\sigma_{t}=9.6 \times 10^{-7} \sim 10^{-6} \mathrm{~s}$. This value is obtained by averaging the precision achieved for the best measured pulsars included in the International Pulsar Timing Array Project, Table 1 in Hobbs et al. (2010). We are aware that only around 40 pulsars are monitored with such accuracy for the time being. Any future improvements in precision and scope would directly translate into an improvement of the results presented in this section.

For each pulsar we have the Galactic latitude $(\phi)$, the Galactic longitude $(\theta)$, and the distance $(L)$ to the Earth. We transform these coordinates to $(\alpha, \beta)$, where $\alpha$, as already explained, is the angular separation between the Earth-GW source line and the Earth-pulsar line. $\beta$ corresponds to the azimuthal angle of the pulsar with respect to the plane perpendicular to the Earth-source line.

The statistical significance of the timing residual will be

$$
\sigma=\sqrt{\frac{1}{N_{p} N_{t}} \sum_{i=1}^{N_{p}} \sum_{j=1}^{N_{t}}\left(\frac{H\left(T_{E}^{i, j}, L_{i}, \alpha_{i}, \beta_{i}, Z_{E}, w, \varepsilon, \Lambda\right)}{\sigma_{t}}\right)^{2}}
$$

where $\sigma_{t}$ is the accuracy with which we are able to measure the pulsar signal period. We take $\sigma_{t}=10^{-6} \mathrm{~s}$ as mentioned. The index $i$ running from 1 to $N_{p}$ labels the pulsars included in the average.

In the statistical average we assume an observation time span of approximately three years, starting at the time the signal is $10^{16} \mathrm{~s}$ old (time of arrival at our Galaxy). We assume that we perform observations every 11 days, that is $N_{t}=101$; $10^{16} \mathrm{~s} \leqslant T_{E} \leqslant 1.00000001 \times 10^{16} \mathrm{~s}$. Since the coalescence times of supermassive black hole binaries can be of the order of $10^{7} \mathrm{~s}$ (Brown et al. 2007; this is a much shorter timescale than the time of arrival of the perturbation to the local system), it is justified to use $T_{E}=\left(Z_{E} / c\right)$. In Figure 3(d) one can also see that the position of the enhancement is not significantly altered in the time span of the observation. While we are aware that three years is a short time (most studies consider observational periods from five to ten years) we only intend to present here a proof of principle and we prefer to consider a short period for our numerical analysis. Longer periods of observation will of course reinforce the signal.

We turn to the angular dependence of the significance. In the following $\sigma(\alpha)$ is plotted keeping $\alpha$ as a free parameter (note that it is not summed), that is, using a set of five fixed pulsars supposed to be exactly at the same angular separation ${ }^{4}$ from a source the position of which we vary between $0 \leqslant \alpha \leqslant \pi$ (the result is again symmetrical for $\pi \leqslant \alpha \leqslant 2 \pi$ ). This could be done for any set of five pulsars, since, as shown in the previous section, the position of the peak does not depend on the values

\footnotetext{
4 The chosen pulsars belong to a globular cluster that in principle cannot be currently timed with the assumed accuracy due to the internal accelerations within the cluster; in this respect this is still a theoretical exercise. We thank the referee for pointing this out to us.
}

Table 1

List of Pulsars Whose $L_{i}$ and $\beta_{i}$ We Used to Calculate $\sigma(\alpha)$ for a Hypothetical Source at Angular Separation $\alpha$

\begin{tabular}{c}
\hline \hline Pulsars from the ATNF Catalogue \\
\hline J0024-7204E \\
J0024-7204D \\
J0024-7204M \\
J0024-7204G \\
J0024-7204I \\
\hline
\end{tabular}

$L_{i}$ and $\beta_{i}$. However, we used the set of real pulsars in Table 1, which are all close to each other at a distance $L \sim 10^{20} \mathrm{~m}$.

It must be borne in mind that although there are over 600 pulsars, making it easy to find clusters with a similar $\alpha$ (albeit possibly with very different values of $L$ and $\beta$ ), the precision with which they are timed can vary widely. The overall magnitude of the significance depends directly on the precision of the measured period as well as on the amplitude of the wave. The results presented in the following may not be entirely realistic due to the uncertainties in these values, but the general features of the analysis would remain unchanged if more realistic values (eventually available) were used:

$$
\sigma(\alpha)=\sqrt{\frac{1}{5 \times 101} \sum_{i=1}^{5} \sum_{j=1}^{101}\left(\frac{H\left(T_{E}^{i, j}, L_{i}, \alpha, \beta_{i}, 3 \times 10^{24}, 10^{-8}, 1.2 \times 10^{9}, 10^{-35}\right)}{10^{-6}}\right)^{2}} .
$$

Lengths are given in meters, and frequencies in $\mathrm{s}^{-1}$. We observe a huge peak at $\alpha \sim 0.19 \mathrm{rad}$ (see Figure 4). If a source is located at such an angular separation ${ }^{5}$ from the average angular position of the five pulsars chosen for observation, the significance could be boosted some 50 times. Let us compare it to the same calculation taking $\Lambda=0$ and redshifted frequency $w_{\text {eff }}=w /(1+z) ; z \sim 0.008$, which is the corresponding redshift for an object $10^{24} \mathrm{~m}$ away, calculated using both matter and energy densities. No peak is observed.

Now we take a list of observed pulsars well distributed in the Galaxy. The angles $(\alpha, \beta)$ are calculated for all of them considering two hypothetical sources of GWs: one located at Galactic coordinates $\theta_{S 1}=300^{\circ}, \phi_{S 1}=-35^{\circ}$ and another located at $\theta_{S 2}=4^{\circ}, \phi_{S 2}=10^{\circ}$. We order the pulsars from the lowest $\alpha$ to the largest and group them in sets of five. We consider 27 sets of 5 pulsars, giving a list of 135 . For each set we calculate the significance

$$
\sigma_{k}=\sqrt{\frac{1}{5 \times 101} \sum_{i=1}^{5_{k}} \sum_{j=1}^{101}\left(\frac{H\left(T_{E}^{i, j}, L_{i}, \alpha_{i}, \beta_{i}, 3 \times 10^{24}, 10^{-8}, 1.2 \times 10^{9}, 10^{-35}\right)}{10^{-6}}\right)^{2}}
$$

\footnotetext{
5 The angular position of the enhancement corresponds approximately to a stationary point of the phase of the wave, i.e., the path along which the phase of the gravitational wave is practically constant, making the integrated timing residual maximal. If one considers the wave front of a Minkowskian spherical wave, there is no path parameterized as the argument in Equation (4), corresponding to a straight line in space, with a constant phase (other than paths pointing to the source, for which the amplitude is zero). Instead, if one considers a wave with a frequency and wave vector depending on the spacetime coordinates, it is in principle possible to find a path where the changes in frequency and wave vector compensate for the change in phase, making the overall phase constant along that path. It can be seen by deriving the phase of the trigonometric function in $h_{i j}$ with respect to $x$ and imposing the stationary phase condition that one gets a solution for $\alpha \neq 0$ that depends very weakly on $x$ itself.
} 

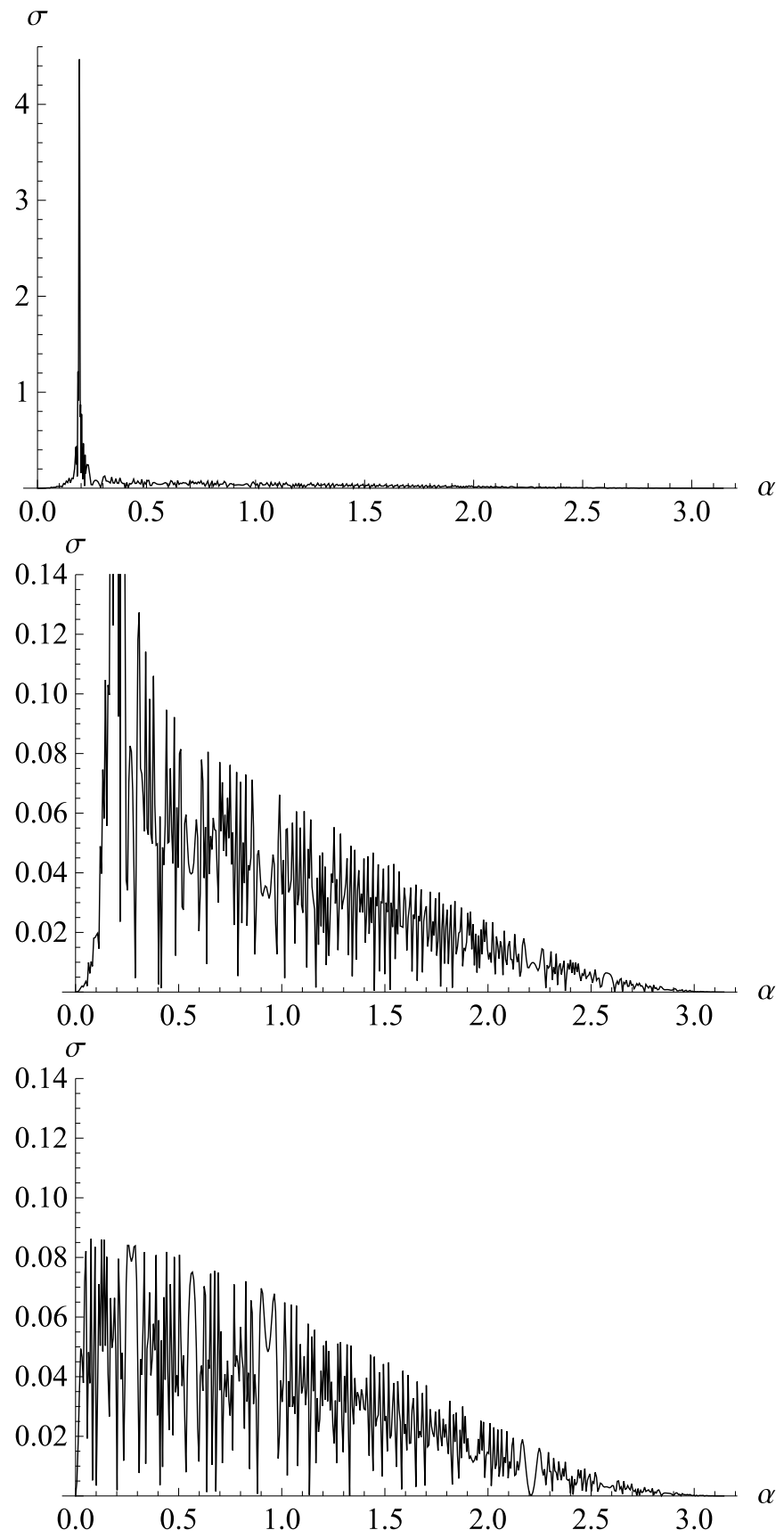

Figure 4. Top: $\sigma(\alpha)$ for $\Lambda=10^{-35} \mathrm{~s}^{-2}$, middle: zoom on the lower values for $\Lambda=10^{-35} \mathrm{~s}^{-2}$, and bottom: comparison to $\Lambda=0$.

and plot it as a function of the average angle of the set, $\bar{\alpha}_{k}=$ $\sum_{i=1}^{5_{k}}\left(\alpha_{i} / 5\right)$ with $1 \leqslant k \leqslant 27$. Note that this is different from Equation (11); here we choose two hypothetical fixed sources and a long list of pulsars grouped by their angular separation $\alpha$ to these sources. This could be a realistic calculation once real sources are considered. The results obtained are plotted in Figure 5. In both cases a very noticeable peak is observed at the expected angle.

The reason why the peak for Source 2 is lower than that for Source 1 is that Source 2 is located close to but not at the precise angular separation of a real cluster of pulsars. This is meant to illustrate that even in that case a significant enhancement of the signal can be achieved.
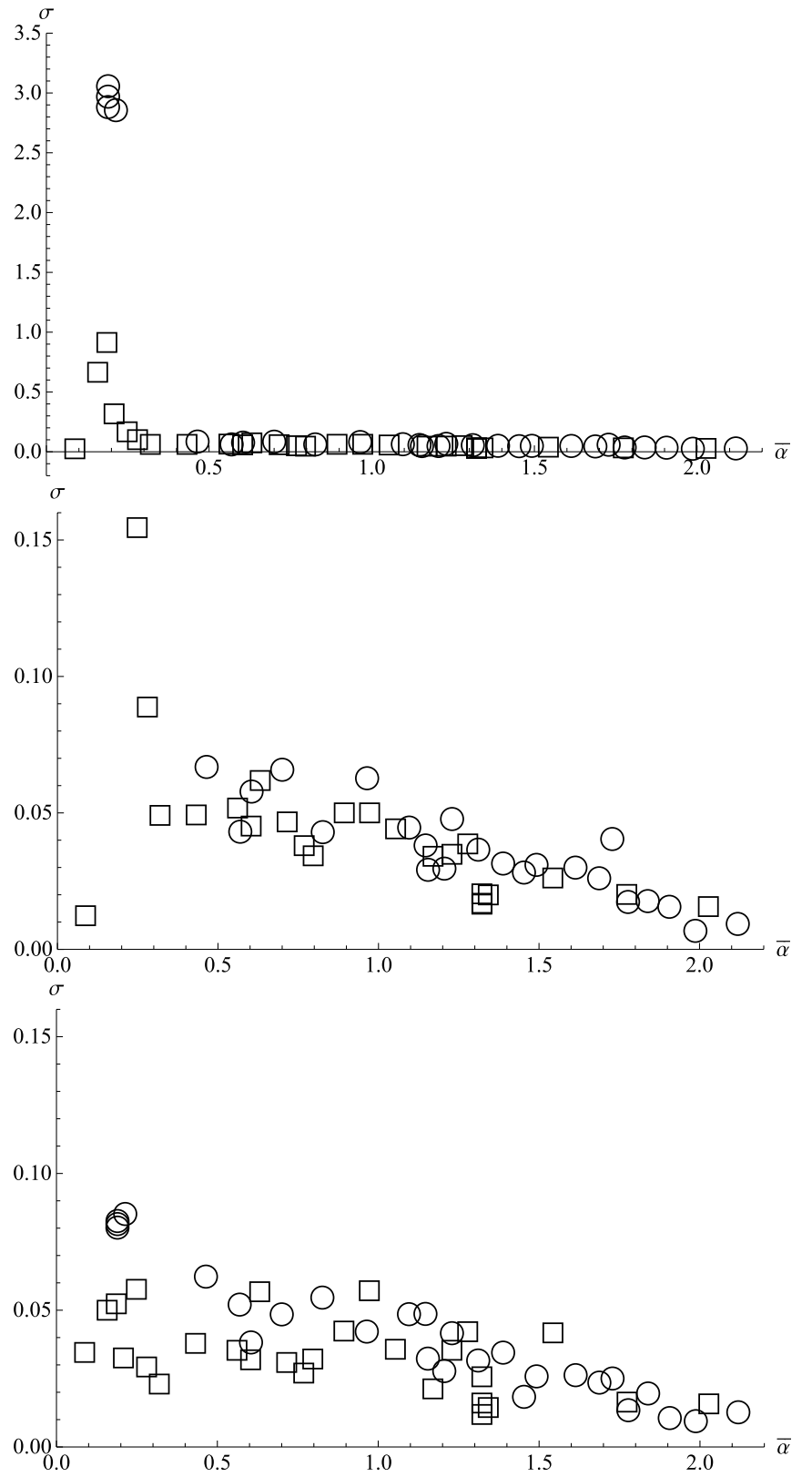

Figure 5. Plot of $\sigma_{k}\left(\bar{\alpha}_{k}\right), k=1,27 . \Lambda=10^{-35} \mathrm{~s}^{-2}$. Circles correspond to Source 1 and squares to Source 2. The full range is shown at the top, and a zoom on the lower values for $\Lambda=10^{-35} \mathrm{~s}^{-2}$ and comparison to $\Lambda=0$ is shown on the middle and bottom, respectively.

Finally, the dependence of $\sigma$ on the frequency

$$
\sigma(w)=\sqrt{\frac{1}{N_{p} \cdot 101} \sum_{i=1}^{N_{p}} \sum_{j=1}^{101}\left(\frac{H\left(T_{E}^{i, j}, L_{i}, \alpha_{i}, \beta_{i}, 3 \times 10^{24}, w, 1.2 \times 10^{9}, 10^{-35}\right)}{10^{-6}}\right)^{2}}
$$

has also been investigated. Some of our preliminary checks indicated that no differences at all were observed in the power spectrum when the value of $\Lambda$ was changed and that, as expected (Hobbs 2008a, 2008b; Hobbs et al. 2010; Jenet et al. 2006), the signal follows a power law $\sigma \sim(1 / w)$. However, let us take a closer look at the dependence on frequency for a short list of pulsars located at the right angular separation to observe the peak. We have already seen that the significance grows notoriously in this angular region. Figure 6 (middle) shows the 

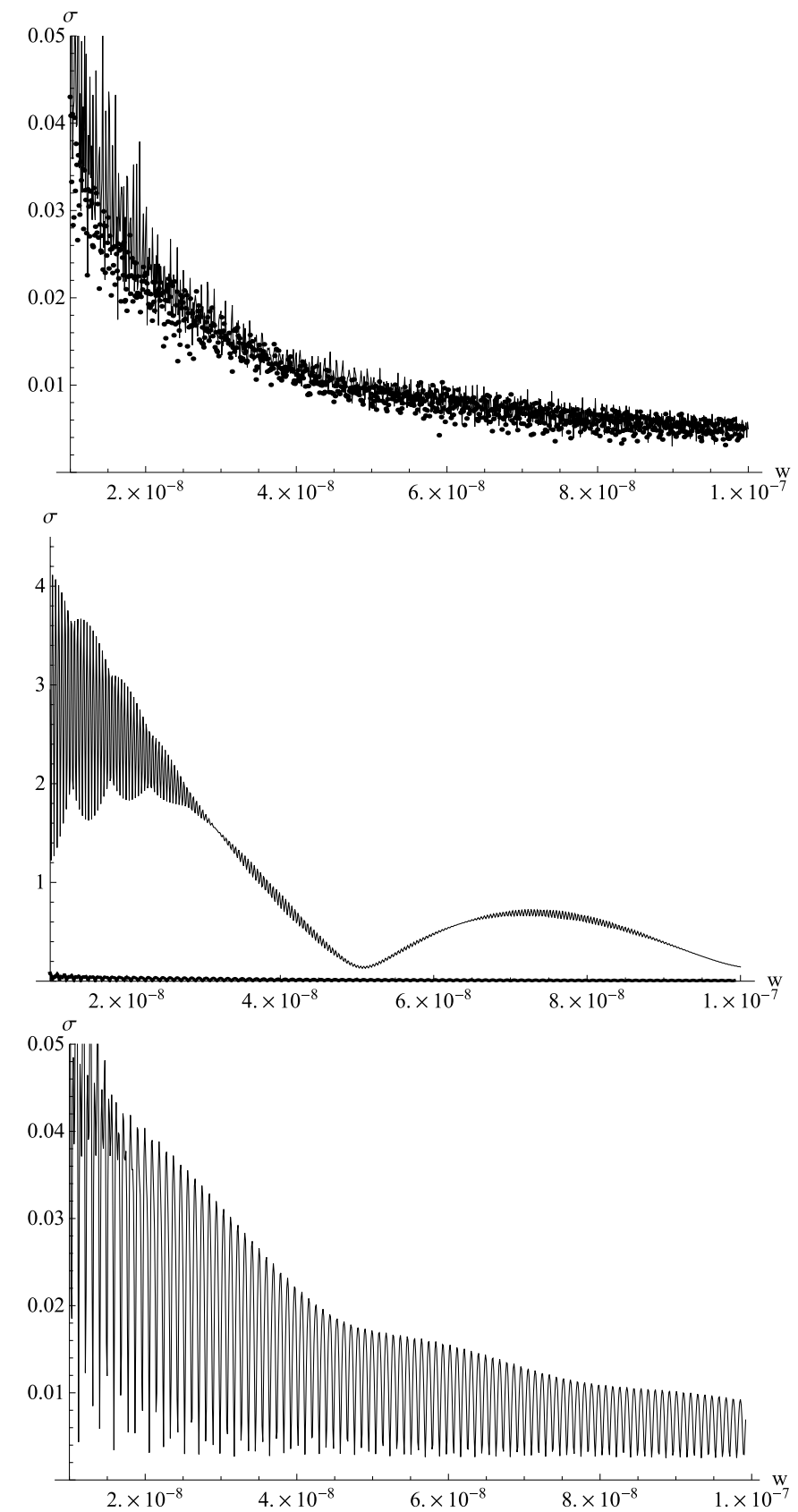

Figure 6. Top: $\sigma(w)$ for 15 pulsars away from the peak angular region for Source 1; the solid line corresponds to $\Lambda=10^{-35} \mathrm{~s}^{-2}$ and dots correspond to $\Lambda=0$. Middle: $\sigma(w)$ for 15 pulsars at the peak angular region for the same source; the solid line corresponds to $\Lambda=10^{-35} \mathrm{~s}^{-2}$ and the data close to the horizontal axis correspond to $\Lambda=0$. Bottom: zoom on the $\Lambda=0$ case.

frequency dependence of the signal for 15 pulsars at the right spot with respect to Source 1 . As can be clearly seen, the signal significance grows enormously again for $\Lambda=10^{-35} \mathrm{~s}^{-2}$ and apparently does not follow a power law. For the same short list of pulsars and for $\Lambda=0$ the signal falls back to smaller values and its envelope shapes toward a power law. In Figure 6 (top) we also present the same plot for 15 pulsars located at an angular separation of around $\alpha \sim 1.1 \mathrm{rad}$, that is, away from the peak. In this case we see no differences between the different values of the cosmological constant, as well as a clear power-law behavior. The magnitude of the signal is compatible with that of the 15 pulsars at the peak separation when $\Lambda=0$.

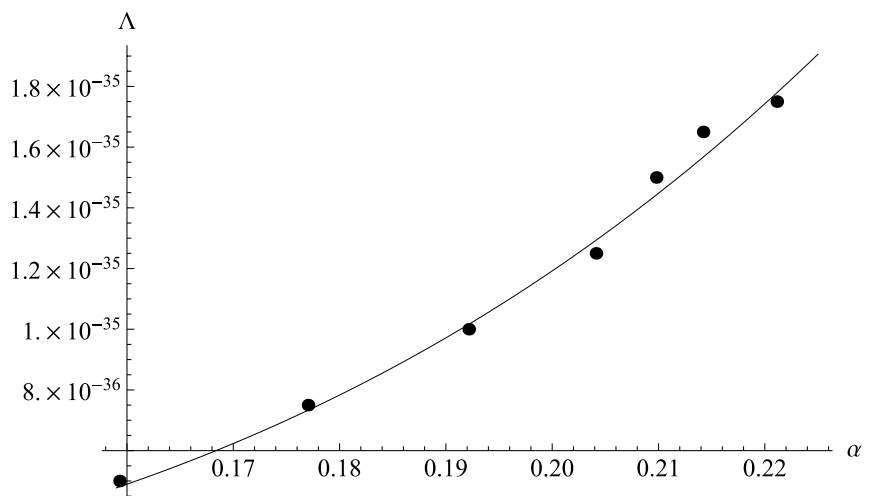

Figure 7. $\Lambda(\alpha)$ obtained numerically from the positions of the peaks in the $\sigma(\alpha)$ plots for different values of the cosmological constant (dots) and obtained analytically from an approximation of the Fresnel functions involved in the timing residual (line).

\section{MEASURING THE COSMOLOGICAL CONSTANT}

We have seen in the previous section that there is an enhancement in the timing residual for a particular value of the angle $\alpha$ when GWs propagating in de Sitter spacetime are measured. Among all the dependences, and when the distance to the source is well known, the most relevant appears to be that related to the value of the cosmological constant $\Lambda$. The position of the peak depends strongly on the value of $\Lambda$, and moves toward the central values of the angle for larger values.

The values of $\Lambda$ as a function of the position at which the peak would be found are plotted in Figure 7 (dots) using the positions found in the plots for $\sigma(\alpha)$ (Equation (11)) for different values of the cosmological constant. This calculation was carried out using two independent numerical methods to ensure that it is free of numerical instabilities (this is a necessary precaution as large numbers are involved).

We argued in Section 2 that the position of the peak is determined by the Fresnel functions one obtains when calculating the timing residuals. Indeed, the integral $I$ in Equation (9), which captures the crucial effect, gives a prefactor times a combination of Fresnel functions times a combination of ordinary trigonometric functions. The last are featureless; however, the prefactor becomes quite large for a specific value of the parameters involved. This particular value renders the Fresnel function close to zero and the product is a number close to 2 . Away from this point the net result is small.

Using the series expansion of the Fresnel functions at first order we are able to obtain an approximate analytical expression for the relation $\Lambda(\alpha)$; that is, for the value of the cosmological constant that (all other parameters being fixed) gives a strong enhancement of the significance $\sigma$ at a given angle $\alpha$

$$
\Lambda(\alpha)=\frac{12 c^{2} \sin ^{4}\left(\frac{\alpha}{2}\right)}{\left(\left(c T_{E}-Z_{E}\right) \cos \alpha+Z_{E}\right)^{2}} \simeq \frac{12 c^{2} \sin ^{4}\left(\frac{\alpha}{2}\right)}{Z_{E}^{2}},
$$

which is also shown in Figure 7 (line). We have used the fact that, taking into account the duration of a black hole merger, $c T_{E} \simeq Z_{E}$. Equation (14) is a clear prediction that could be eventually tested. In fact, this effect could also facilitate enormously the detection of GWs coming from massive binary black holes by carefully selecting and binning groups of pulsars, although the possibility of measuring $\Lambda$ locally certainly looks to us more exciting.

Throughout this work we have considered only the effect of $\Lambda$ on GWs and the way they affect pulsar timing residuals, and we 
have neglected the effect of matter or matter density. In fact, the main effect of the latter would be through the familiar redshift in the frequency of the GW. The overall frequency value does not play a crucial role in the previous discussion provided it is low enough to be detectable in the PTA. It is its dependence on the space-time coordinates that brings about new effects. It is probably useful to remind the reader that $\Lambda$ is assumed to be an intrinsic property of spacetime, present at all scales. It would be easy to implement more realistic models in our study, if reasonably well-defined ones were available. In fact, these uncertainties constitute strong reasons to try to measure $\Lambda$ locally.

\section{SUMMARY}

The purpose of this work was to investigate the local effects of the cosmological constant for the detection of GWs in PTAs. The GW function is usually modeled as a massless wave, either plane or spherical, traveling in flat spacetime. The expansion of the universe is accounted for by including a redshift in the frequency. Major problems are related to modeling the source and assessing the strain of the amplitudes of the waves. Here we obviate these by just assuming a spherical wave and focus on the fact that the waves propagate in a de Sitter spacetime rather than in a flat spacetime.

We use a wave solution previously derived in FRW coordinates, which we expect to be more realistic than the redshifted usual waves. With this, we calculate the timing residuals induced in the signal of known pulsars in our Galaxy, predicting a particular value of the angle subtended between the source and the pulsar where an enhanced significance of the timing residual is observed. We argue that the position of this peak depends strongly on the value of the cosmological constant. This peak is absent when the calculations are carried out with usual, Minkowski solution, redshifted waves. We propose two hypothetical sources at two distinct positions for which we calculate the timing residuals' significance using a real set of pulsars. The peak is observed at the predicted angular position. Finally, we obtain the angular dependence of the value of the cosmological constant using the position of the peak for different values of $\Lambda$ and analytically from the Fresnel functions involved in the calculation. This could represent an independent method to determine the value of the cosmological constant.

We should finish with a disclaimer. The results presented in this paper are by all means preliminary. We have addressed the somewhat academic study of an isolated point-like source of GWs and proceeded to analyze its influence on PTAs. In fact, we should expect a complete background of sources. Preliminary studies indicate that single-source detection may be feasible, but at values of redshift larger than those considered here (Hobbs et al. 2010). Of course these studies do not consider the effects of $\Lambda$ discussed in this paper. In addition, a full error analysis, including all unknowns in the appropriate covariance matrix, should be performed before drawing conclusions on the statistical significance of the effect. Some of these studies are only feasible by the PTA collaborations themselves whose interest in the effect presented here we hope to have aroused.

We acknowledge the financial support from research grants FPA2007-20807, SGR2009SGR502. This research is supported by the Consolider CPAN project. We thank Ll. Garrido, J. Bernabeu, J. M. Paredes, J. Salvadó, and J. Moldón for useful discussions on the subject. We also thank L. Verde for reading the manuscript and A. Sintes for extended discussions and for pointing us to several references.

\section{REFERENCES}

Adkins, G. S., McDonnell, J., \& Fell, R. N. 2007, PhRvD, 75, 064011

Balaguera-Antolinez, A., Boehmer, C. G., \& Nowakowski, M. 2006, CQGra, 23,485

Bernabeu, J., Espinoza, C., \& Mavromatos, N. E. 2010, PhRvD, 81, 084002

Bernabeu, J., Espriu, D., \& Puigdomènech, D. 2011, PhRvD, 84, 063523

Brown, D. A., Crowder, J., Cutler, C., Mandel, I., \& Vallisneri, M. 2007, CQGra, 24, S595

Deng, X., \& Finn, L. S. 2011, MNRAS, 414, 50

Eisenstein, D. J., Zehavi, I., Hogg, D. W., et al. (SDSS Collaboration) 2005, ApJ, 633,560

Fu, L., Semboloni, E., Hoekstra, H., et al. 2008, A\&A, 479, 9

Guzzo, L., Pierleoni, M., Meneux, B., et al. 2008, Natur, 451, 541

Hobbs, G. 2008a, CQGra, 25, 114032

Hobbs, G. 2008b, JPhCS, 122, 012003

Hobbs, G. 2011, High-Energy Emission from Pulsars and their Systems (Berlin: Springer), 229

Hobbs, G., Archibald, A., Arzoumanian, Z., et al. 2010, CQGra, 27, 084013

Iorio, L. 2008a, AdAst, 2008, 268647

Iorio, L. 2008b, NewA, 14, 196

Jenet, F. A., Hobbs, G. B., van Straten, W., et al. 2006, ApJ, 653, 1571

Kottler, F. 1918, AnPhy, 361, 401

Kowalski, M., Rubin, D., Aldering, G., et al. (Supernova Cosmology Project Collaboration) 2008, ApJ, 686, 749

Lee, K. J., Wex, N., Kramer, M., et al. 2011, MNRAS, 414, 3251

Linder, E., \& Perlmutter, S. 2007, PhyW, 20, 24

Manchester, R. N., Hobbs, G. B., Teoh, A., \& Hobbs, M. 2005, AJ, 129, 1993

Peebles, P. J. 1976, Ap\&SS, 45, 3

Peebles, P. J. 1980, The Large Scale Structure of the Universe (Princeton, NJ: Princeton Univ. Press)

Perlmutter, S., Aldering, G., Goldhaber, G., et al. (Supernova Cosmology Project Collaboration) 1999, ApJ, 517, 565

Price, R. H., \& Yang, Y. 2008, AmJPh, 76, 930

Riess, A. G. 2000, PASP, 112, 1284

Riess, A. G., Filippenko, A. V., Challis, P., et al. (Supernova Search Team Collaboration) 1998, AJ, 116, 1009

Riess, A. G., \& Livio, M. 2006, ApJ, 648, 884

Riess, A. G., Strolger, L.-G., Tonry, J., et al. (Supernova Search Team Collaboration) 2004, ApJ, 607, 665

Rindler, W. 1998, PhLA, 245, 363

Rindler, W. 2006, Relativity: Special, General, and Cosmological (2nd ed.; Oxford: Oxford Univ. Press)

Seo, H. J., \& Eisenstein, D. J. 2003, ApJ, 598, 720

Sereno, M., \& Jetzer, P. 2006, PhRvD, 73, 063004

Spergel, D. N., Bean, R., Dore, O., et al. (WMAP Collaboration) 2007, ApJS, 170,377

Suto, Y. 1993, PThPh, 90, 1173

Suto, Y. 1996, in Current Topics in Physics: Proc. of the Inauguration Conf. of the Asia-Pacific Center for Theoretical Physics, ed. Y. M. Cho, J. B. Hong, \& C. N. Yang (Seoul: Seoul National Univ.), 734

Wood-Vasey, W. M., Miknaitis, G., Stubbs, C. W., et al. (ESSENCE Collaboration) 2007, ApJ, 666, 694 